Title: Schedule performance measurement based on statistical process control charts

Authors: Omogbai Oleghe1, Konstantinos Salonitis ${ }^{2}$

\title{
Affiliation:
}

Manufacturing and Materials Department

Cranfield University

MK43 0AL, Cranfield, UK

1: email: o.a.oleghe@cranfield.ac.uk

2: Tel: +44 (0) 1234 758347, email: k.salonitis@cranfield.ac.uk (corresponding author)

\section{Bibliographical Statements}

\section{$\underline{\text { O. Oleghe }}$}

Mr. Omogbai A. Oleghe has received his first degree in Mechanical Engineering graduating in 1991 from the University of Lagos, Nigeria. He also holds a Masters in Business Administration (MBA) from the Birmingham Business School, University of Birmingham, England where he graduated with a distinction in 1996. He recently concluded another Masters program in Manufacturing Consultancy at the School of Applied Sciences, Cranfield University, England, in September 2013.

He is Managing Director of Rite Pak Company Limited, a plastics film print and packaging company. His professional experience spanning 21 years covers operations management within the manufacturing sector.

\section{$\underline{\text { K. Salonitis }}$}

Dr Konstantinos Salonitis is a Lecturer in Manufacturing Systems in Cranfield University. He graduated from University of a Patras with a Diploma in Mechanical Engineering in 2001 and was awarded a PhD on Manufacturing Processes in 2006. He has worked as a Research Engineer at the University of Patras in a number of research projects funded by the CEU. He has participated in more than 10 projects as Project Manager or Technical Site Manager. Dr Salonitis joined Cranfield University in February 2012; he is the Manufacturing and Management Programme director. He has published more than 100 research papers in major international journals and internationally referred conferences. 


\title{
Schedule performance measurement based on statistical process control charts
}

\begin{abstract}
In a job-shop manufacturing environment, achieving a schedule that is on target is difficult due to the dynamism of factors affecting the system, and this makes schedule performance measurement systems hard to design and implement. In the present paper, Statistical Process Control charts are directly applied to a scheduling process for the purpose of objectively measuring schedule performance. SPC charts provide an objective and timely approach to designing, implementing and monitoring schedule performance. However, the use of Statistical Process Control charts requires an appreciation of the conditions for applying raw data to SPC charts. In the present paper, the Shewart's Individuals control chart are applied to monitor the deviations of actual process times from the scheduled process times for each job on a process machine. The Individuals control charts are highly sensitive to non-normal data, which increases the rate of false alarms, but this can be avoided using data transformation operations such as the Box-Cox transformation. Statistical Process Control charts have not been used to measure schedule performance in a job shop setting, so this paper uniquely contributes to research in this area. In addition, using our proposed methodology enables a scheduler to monitor how an optimal schedule has performed on the shop floor, study the variations between planned and actual outcomes, seek ways of eliminating these variations and check if process improvements have been effective.
\end{abstract}

\section{Keywords}

Performance measurement system, job shop scheduling, schedule performance, statistical process control charts, individuals controls chart. 


\section{Introduction}

Performance measurement can be defined as a means of quantifying the effectiveness of past actions with a view to improving present and future outcomes (Neely et al, 2012). The importance of performance management systems (PMSs) cannot be overemphasized as results suggest that high performing organizations rely on a wider set of PMSs than low performing organizations (Henri, 2006). In practice, implementing and monitoring a PMS is fraught with challenges such as inclusion of subjectivity into the PMS and a lack of continuous improvement, so a PMS that tackles these issues is a prerequisite for the success of the PMS.

A schedule is a plan for when an activity will begin and when it will end, and it enables firms deploy resources in a timely and efficient manner. Designing a production schedule in a manufacturing system requires organizations to appraise a multitude of inputs. In a job-shop manufacturing system characterised by low volume, high variety customised products, scheduling is a complex problem. Scheduling techniques such as those based on dispatching rules and genetic algorithms, aim to find an optimal schedule. However, Ouelhadj and Petrovic (2009) proved that while an optimal schedule may be derived at a stage in the cycle of activities, it might become un-optimal at any time due to a variety of reasons such as machine breakdown or rush orders, and this has led to a gap between theory and practice. Researchers have attempted to counter this problem by using rescheduling strategies to correct the schedule.

Schedule performance evaluation is a comparison between the actual outcome and the schedule or plan. Typical schedule performances metrics are make span, flow time, tardiness, set up time, work in process and machine utilization. In practice these measures, with a multitude of other performance measures, are in conflict within an organisation, and choosing one or a set over others can be demanding. De Snoo et al.(2011) carried out a survey where they interviewed 86 managers and planners in 43 organizations, to establish the criteria they use in measuring schedule performance. In that survey, some respondents commented that schedule error could be eliminated if schedules contained the correct process time, while some focused on the importance of the availability, reliability and completeness of information. 
Statistical process control (SPC) charts first introduced by Shewart in the 1930s are used to document variations in a quality characteristic within the process by distinguishing common predictable causes of variation from special, unpredictable causes. SPC charts were originally designed and used to monitor and improve product quality by monitoring the production process. However, its usage is now been extended to cover many organizational processes within manufacturing and service operations. MacCarthy and Wasusri (2002) detail a broad range of SPC chart usage in non-standard applications. They found that approximately $74 \%$ of researchers in that domain used the Shewart control charts, and as much as $80 \%$ used SPC charts to monitor and evaluate a process. SPC charts provide a simple approach to performance measurement through process monitoring. Morgan and Dewhurst (2007) used SPC charts to measure and compare the performance of suppliers to a supermarket chain, while Grygoryev and Karaptrovic (2005) applied SPC charts as a performance measurement tool to measure teachers' contribution to student knowledge.

Using SPC charts in non-conventional environments poses a number of challenges. Applying SPC charts to a process requires an appreciation of the underlying criteria that the data must exhibit, and an understanding of the process that is to be monitored. These criteria require that the data must: (a) be independent, (b) approximate a known distribution and (c) be sequential in time (Slavin, 2006). Many real life processes are rarely defined by these criteria, and recent research efforts are succeeding in their effort to counter these assumptions, by modifying the traditional Shewart charts and by transforming non-normal data to normal data. Control charts, and in particular the IX charts, are highly sensitive to non-normal data. When non-normal data is plotted, the rate of false alarm is increased (Chen and $\mathrm{Su}, 2005)$. A false alarm occurs when a falsehood is believed to have happened, when in actual fact there is none. Performing data transformation on a set of data simply involves performing the same mathematical operation on each value in the data set, so that the values are usable for the purpose the data is collected. In SPC chart applications, data transformation takes the form of converting a set of non-normal data values to one that is normal or with an approximately normal distribution. 
Within the present paper we aim to apply SPC tools to a scheduling system of five similar machines or sub-processes, for the purpose of objectively and continuously measuring schedule performance and minimizing schedule error. The quality parameter that will be measured is the deviation of actual process time from planned process time for each job order. The IX chart is sufficient for this purpose since the data comes from individual observations. Because the IX chart is highly sensitive to non-normal data, we will test and correct the data to ensure normality. We will also check the within and between subprocess data dependency. The IX charts will be used to document the process variations and Pareto charts will be used to support our analysis with the aim of minimizing schedule error. The control limits of the IX charts will be used as performance targets so that an effective PMS is set up for the system.

\section{Schedule performance measurement using SPC charts}

There is no agreed definition of what constitutes scheduling performance (De Snoo et al., 2011), and in practice, scheduling performance is viewed is different ways. However a common notion is that scheduling performance should be a measure of the execution of the schedule. There are numerous papers that have been put forward, where SPC charts are deployed within a PMS. Coleman et al (2001) used SPC charts in the development of a safety measurement framework. They used the control charts to signal warning limits that act as action limits, giving guidelines for expected performance. Rungasamy et al. (2002) carried out a survey of 33 manufacturing small and medium enterprises in the UK. They identified some critical success factors (CSF) in the implementation of SPC in the firms. Amongst these CSFs were measurement system evaluation and identification of critical quality characteristics for the system. Fullerton (2003) studied how SPC charts are used within performance measurement techniques in her survey of 447 US manufacturing firms, and found that on-time delivery ranked highest in their performance measures. Della (2000) presented how SPC has enabled Harley Davidson improve on product quality by integrating SPC charts into their automated processes. In the Harley Davidson case, the SPC charts monitor the process by using color-coded signals on the 
SPC chart to flag off danger points. This enables them to react to the process before the process yields products that are out of specification.

Amongst the few researchers that have directly applied SPC charts to scheduling systems are MacCarthy and Wasusri. In one of their papers they proposed that production planning, scheduling and control should be looked at as a dynamic control process, and SPC techniques could be applied in order to detect all changes in the process (Wasusri and MacCarthy, 2001). They argued that prevention of special causes (through investigation using control charts) is better than quickly taking action in order to ensure a job is not behind schedule. They applied SPC charts to two different companies, one with make-to-stock (MTS) and another that applies both MTS and make-to order (MTO) planning and scheduling strategies. They found out that delay in materials in the first company caused an out of control situation at a given time, while in the second company, the chart showed that the process was not in control and therefore unstable and unpredictable. They summarized in their findings that: control charts enable management appreciate the nature of the company's production planning, scheduling and control process and; the disturbances which affect the efficient execution of the company's production schedules can be found and their effects made clearer with a view to eliminating or managing them. In another paper to address the problem of correlated data, MacCarthy and Wasusri (2001) used SPC to monitor schedule performance in a single machine scheduling system. They applied the Exponentially Weighted Moving Averages (EWMA) control chart on correlated data to monitor flow time in a simulated environment. They summarized that applying SPC to monitor time-related quality characteristics within a scheduling system poses statistical hurdles, which can be surmounted using advanced control charts.

Data correlation is highly evident in job schedules. Correlation is a measure of how strongly related a set of variables are to each other and is a broader definition of data dependency which shows that one data is dependent on the measure from another. Schedules are chronologically timed events of activities, where time of an activity is highly dependent on the time from the previous activity or activities. A schedule outcome such as a delay will cause a delay in the next activity or subsequent activities. This 
peculiar problem in schedules makes statistical charting of a scheduling process to be difficult, as one on the pre-requisites for usable data in SPC charts is that the data must be independent, the rational being that there should be no bias in the data. To counter this problem, one can either use data that is not correlated or apply a time series model such as the Autoregressive Moving Average (ARMA) to the correlated data. ARMA like other time series models predicts future values based on the pattern of present values. The difference between the actually observed values and the ARMA values are taken as residuals, and these residuals are plotted on standard Shewart charts with a high confidence that the data is independent or non-correlated. Time series models are estimated from the data, so there may be errors in estimation, leading to the wrong use of a model (Apley, 2002). Choosing an appropriate ARMA time series model is an ambitious task, which requires a minimum level of statistical expertise to implement and interpret.

There is evidently a large body of research available where SPC is used within a PMS context, but there is sparse research in the application of SPC within a PMS for a scheduling process. SPC has not been used to document schedule error.

\section{The proposed approach}

A process approach to organizational performance is considered to be the panacea for organizational success, as activities are registered in processes (Stanislava, 2012). A clear understanding of a system's scheduling process is key to designing a PMS for it, as a process that is not understood cannot be analysed for improvement (Kueng, 2000).

By analysing historical and current data, decision makers are better placed to take informed decisions (Stanislava, 2012). Measuring the quality of production performance requires an appropriate selection of the performance metrics that will be used to monitor it (Stanislava, 2012). It is more efficient to choose a performance metric from an existing set of generic ones than to formulate new ones (Kueng, 2000). Some performance metrics relating to job scheduling include flow time, set up time and process time. Process time is an important parameter in scheduling as it determines the duration of the activity or process, and scheduling error can be eliminated or minimized if schedules contained the 
correct process time (De Snoo et al, 2011). Accurately implemented schedules will have actual process time and planned process time being equal. The performance metric proposed for an application in a scheduling PMS is the difference between the actual process time and the scheduled process time for each job, taken as a deviation to eliminate negative values resulting from jobs that are ahead of schedule. This deviation can be taken as the actual value or observation, and analysed on the IX chart to monitor the performance of the scheduling process.

An outline of the proposed methodology is presented in the flowchart in Figure 1 and described hereafter. The methodology proposed is based on the use of the Shewart's Individuals (IX) control chart. The Box-Cox transformation technique and probability plots are used to ensure the data set are normally distributed. Pareto charts are applied to the methodology to support the analyses of the Ix chart and aid in improving the process.

The use of the IX chart requires that the data set that is used to plot the control chart be normally distributed. Using the Box-Cox transformation technique for statistical analyses is a method adopted to check for normality and also to transform a non-normal data set to a normally distributed one. A probability plot which also checks normality in a data set is performed on the Box-Cox transformed data to confirm that it is normally distributed, giving us confidence that it can be used to plot the Ix chart.

The IX chart is plotted using a batch of twenty consecutive observations and each observation is marked on the chart. The initial CLs are calculated from the data set and these CLs are used for our analyses. The process is in-control when all observations fall within the CLs, and out of control when one or more observations lie outside of the CLs. Subsequent observations are plotted on the same chart without changing the CLs.

For SPC to be effective, two or more SPC tools should be combined. The Pareto chart tool is used to support the analyses of the IX chart, by identifying the major causes of abnormal variations as well as out-of-control observations. When causes of abnormal variations are identified, they can be eliminated, to ensure they do not occur in the process again. The CLs remain unchanged during process monitoring. They are modified only when there is a sustained and prolonged change in the process performance. 


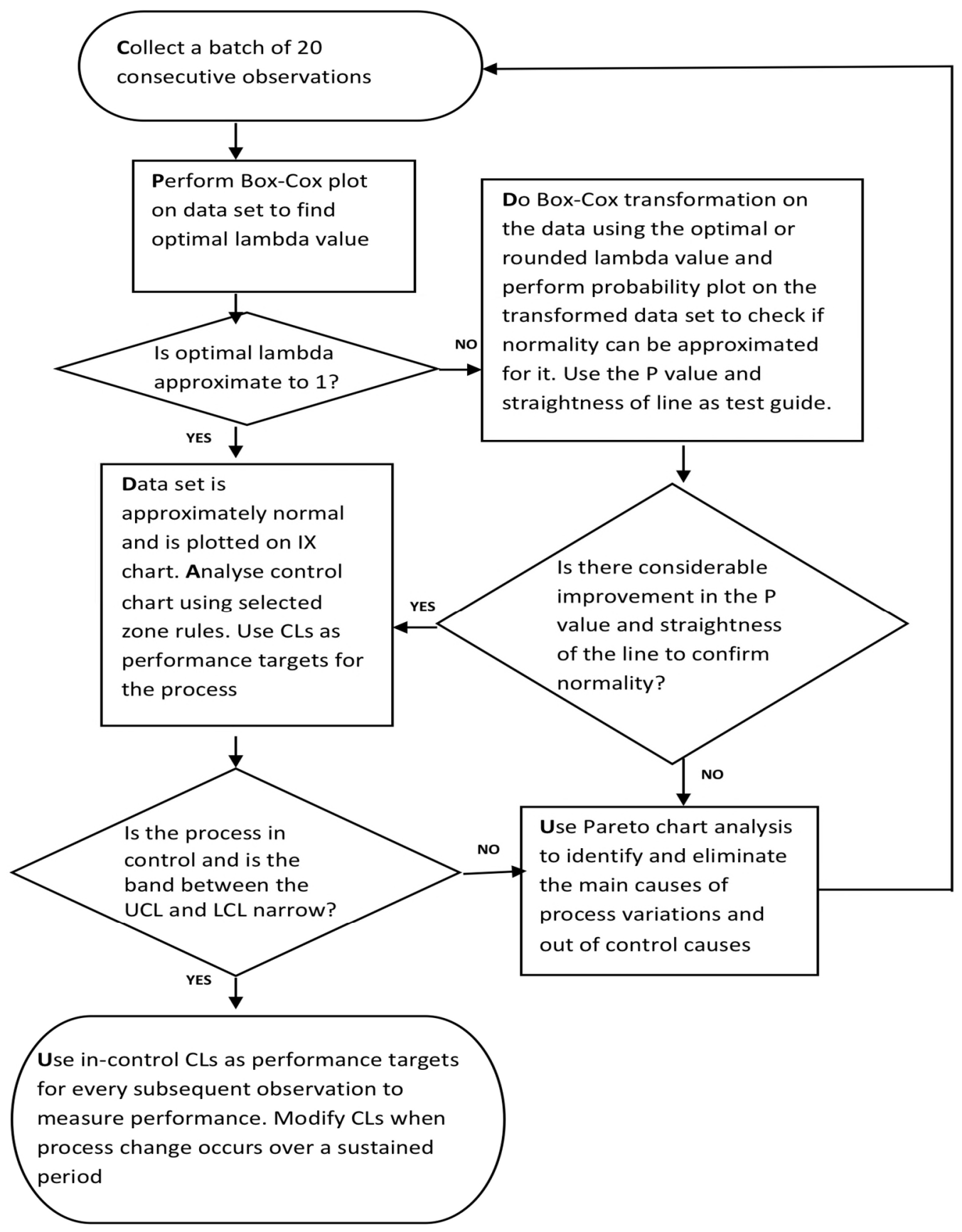

Figure 1 Flow chart of steps in experimental methodology

\section{Implementation and Validation}

In the present paper, the proposed method was applied to the scheduling process of a set of five printing machines in a flexible print packaging factory, herein referred to as the sub-processes. Some jobs can be processed on any machine, while others are machine 
specific. All jobs are allocated to machines on a first-come-first-serve basis, and where similarities in set-up for jobs exist, a dispatching rule based on identical set-ups is used. The five printing machines in the system perform similar operations but differ in their specifications and in their scheduling process. An average of about fifteen jobs are processed daily on the five machines in this department. A simple data collection sheet was used to record every observation for each machine. The factory system is set up for short production runs and only few jobs are processed daily. Every jobs' process time can be easily observed, thereby improving the reliability of the data and eliminating sampling error. About 20 observation samples are needed to develop control chart limits (Wadsworth et al, 2002) and obtain meaningful information from the chart.

As already mentioned, usable data for SPC charts must pass certain tests of normality and independency. In the scheduling process being studied, the process time for each job is independent of the process time of the preceding job; even though it may be argued that there exists some element of dependency arising from similar set-ups or machine wear from previous usage. Where a job was dispatched according to set up, the process time is adjusted by accounting for the time saved resulting from the set up. Additionally, the nature of the manufacturing system enables mainly short production runs of many nonsimilar jobs, and the percentage of jobs scheduled according to a set-up dispatching rule is less than $10 \%$. Machine wear can be argued to cause data dependency as a machine degrades as it is used to process previous jobs. However this dependency is very insignificant as the possibility of the machine degrading substantially to the point where it affects job-to-job output is rare. In addition, a considerable degradation of the machine: will most likely show up as a system shift; will necessitate a re-calculation of chart control limits (Schaeffers and Stephen, 2013); and would likely affect a batch of many jobs rather than degrade and affect job-to-job performance. The autocorrelation plot and scatterplot matrix charts in Figures 2 and 3 respectively confirmed our assumptions. In Figure 2 we see that all lags fall within the confidence limits (dotted lines) and there is no apparent pattern that may suggest correlation. In Figure 3, the amorphous nature of the scatter plots between sub-processes led us to the conclusion that there is no cross correlation amongst the sub-processes. 


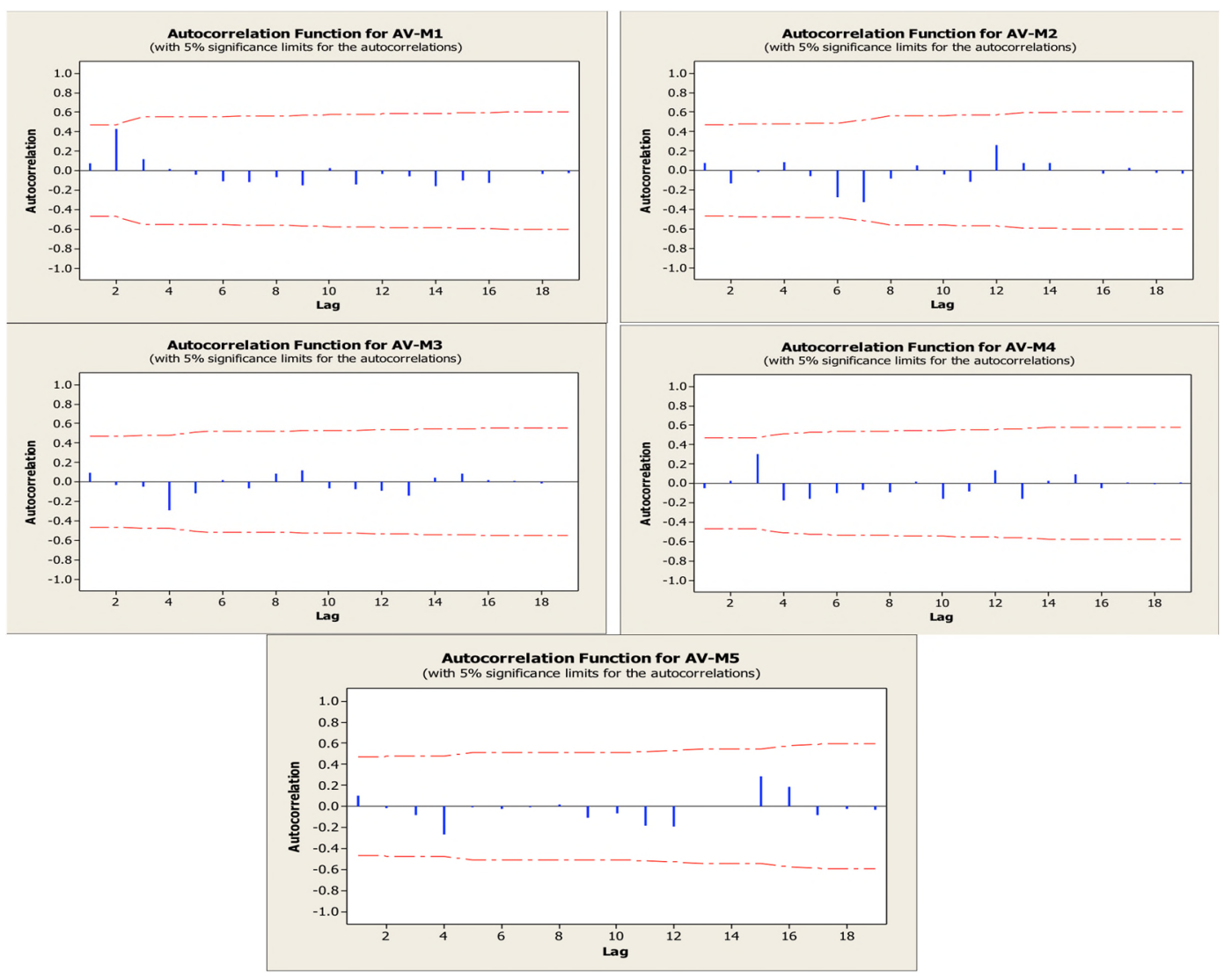

Figure 2 Autocorrelation plot of each machine

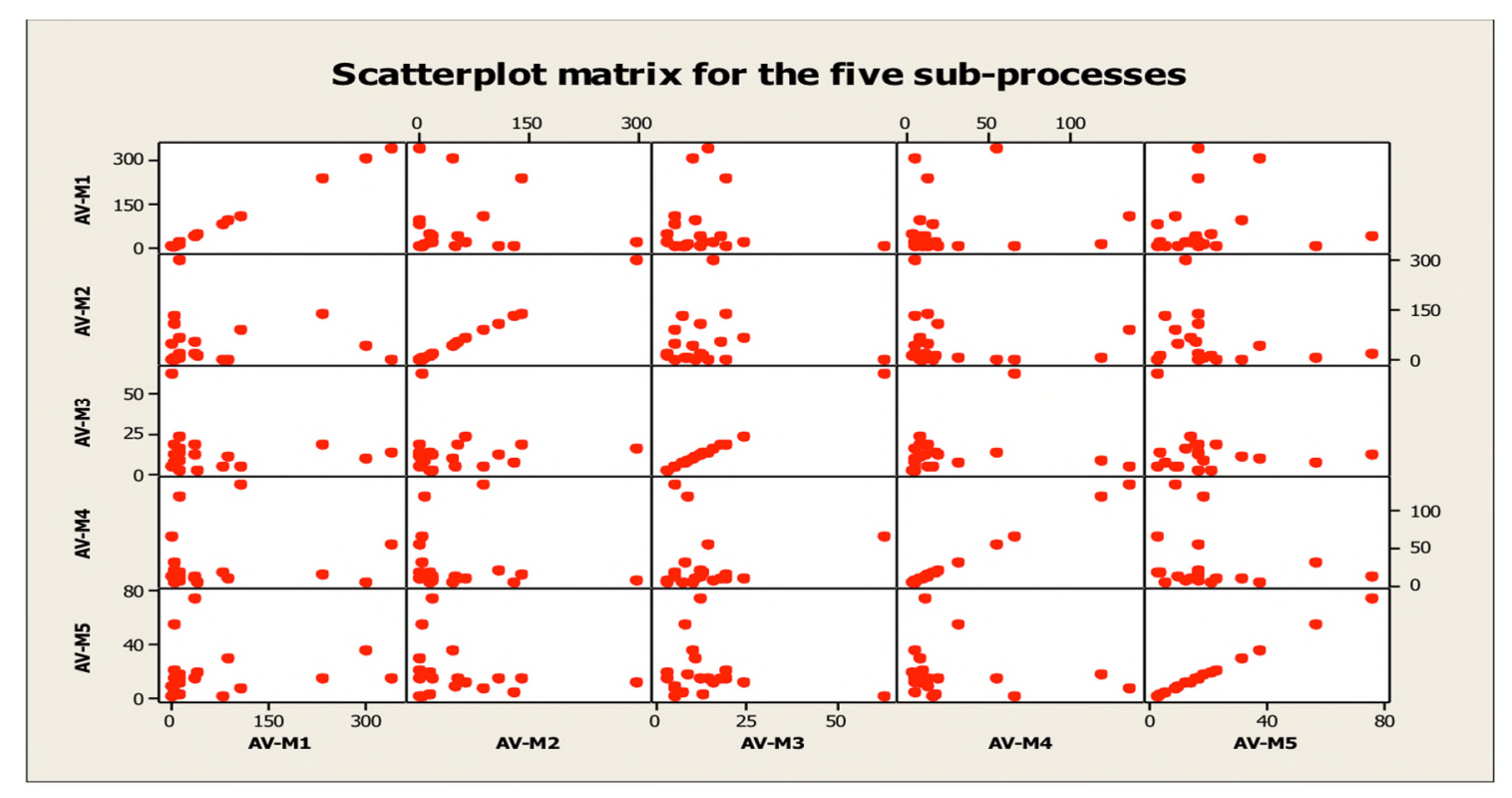

Figure 3 Scatterplot matrix for all the five machines 


\section{Checking data for normal distribution}

Data normality was checked using the Box-Cox transformation tool. A quick way to perform this is by assuming non-normality for all the data, perform a Box-Cox plot of the data set and then use the displayed rounded $\lambda$ value to transform the data. A rounded $\lambda$ that is equal to 1 shows that the data is normally distributed. Figure 4(a) to (e) shows the Box-Cox plot for each machine scheduling data set, and Table 1 is the mathematical operation that is used to transform the batch of non-normal data to a data set with normal distribution for each of the machines' scheduling process.

The Box-Cox transformation is not a guarantee for normality and it is necessary to confirm normality using a probability plot (Buthmann, 2010). Probability plots can be drafted for each machine scheduling data to compare the original data with the transformed data (Figure 5). The straightness of the plot is an indication of normality and with the Box-Cox transformation there is a noticeable improvement on the straightness of the plot for the transformed data set. In addition, the probability plots of the transformed data show a considerable improvement in the $P$ values. The $P$ value determines if the null hypothesis should be rejected or not (for normality in this case) and typically a data set showing a $P$ value less than or equal to 0.05 can be rejected for normality, at $95 \%$ confidence interval.

When we fitted other known distributions such as the Lognormal, Weibull and Exponential distributions to our data, we found that none of these could model the data to a high confidence of fit and none gave a better $P$ value than those obtained using the Box-Cox transformation. Based on this comparison, we chose the Box-Cox transformation to convert our daw data.

Table 1. Mathematical operation using Box-Cox transformation rounded $\lambda$ to convert the actual values in the data set to approximately normal distribution.

\begin{tabular}{ccl} 
Machine No. & $\begin{array}{c}\text { Rounded } \\
\text { Lambda }\end{array}$ & $\begin{array}{l}\text { Mathematical operation corresponding to rounded } \\
\text { Lambda }\end{array}$ \\
\hline 1 & 0 & Logarithm of actual value \\
2 & 0 & Logarithm of actual value \\
\hline
\end{tabular}




\begin{tabular}{lll}
\hline 3 & 0 & Logarithm of actual value \\
4 & -0.5 & $1 \div$ square root of actual value \\
5 & +0.5 & Square root of actual value \\
\hline
\end{tabular}
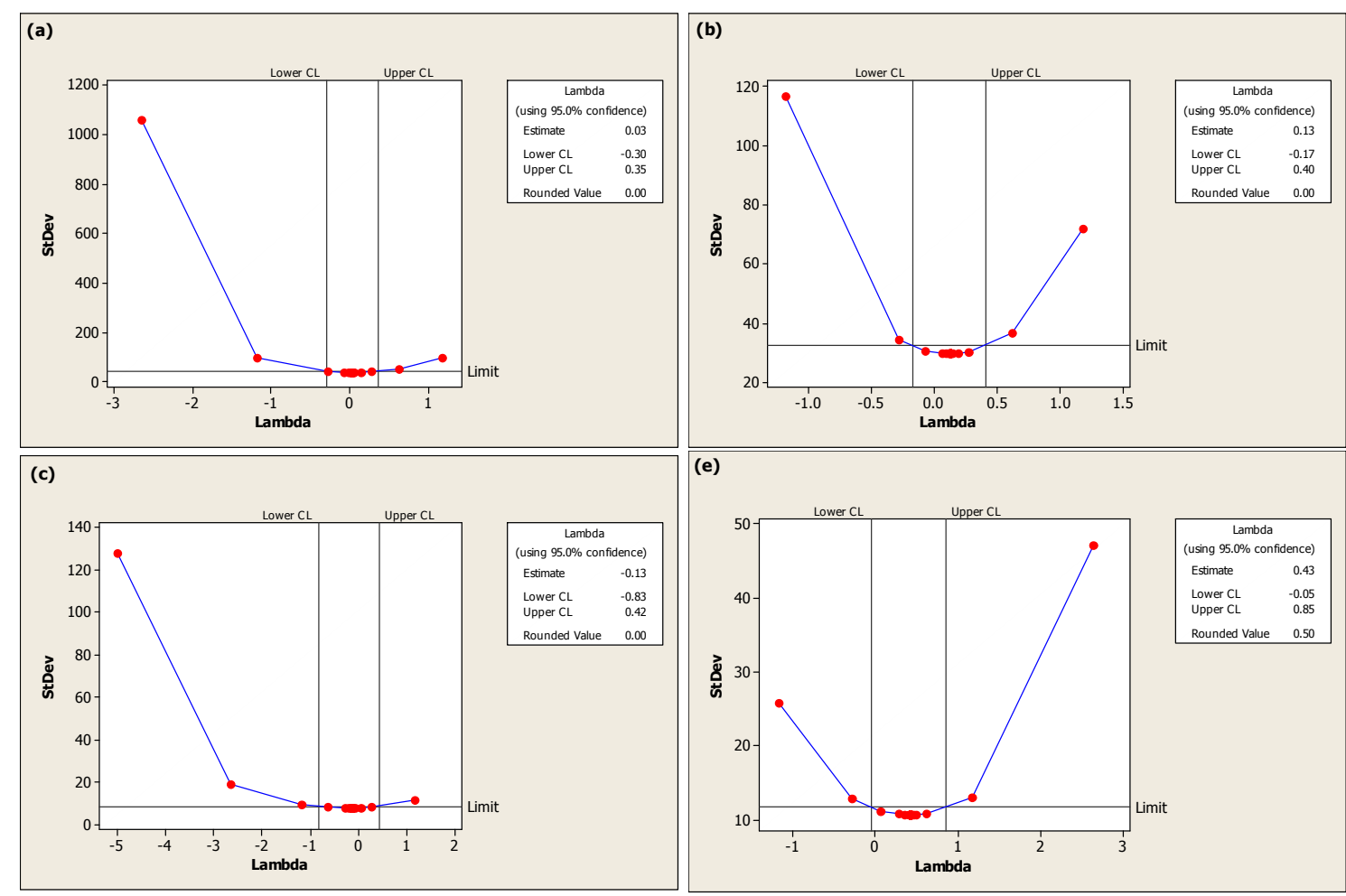


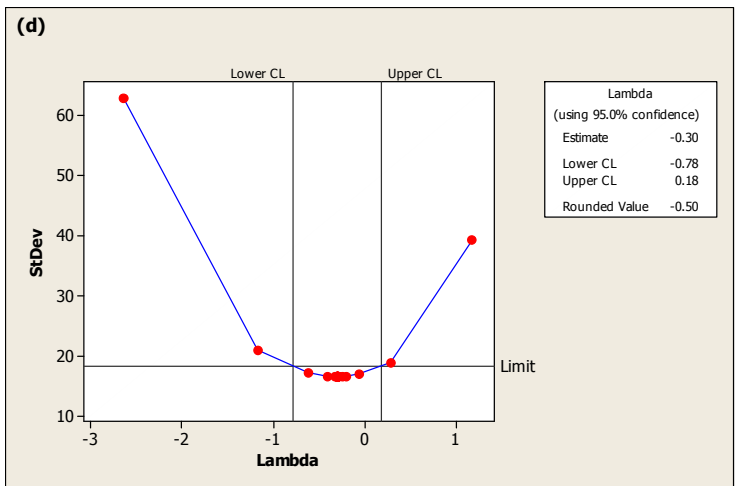

Figure 4. Box-Cox data plot for actual values: (a) machine 1, (b) machine 2, (c) machine 3, (d) machine 4 and (e) machine 5 .
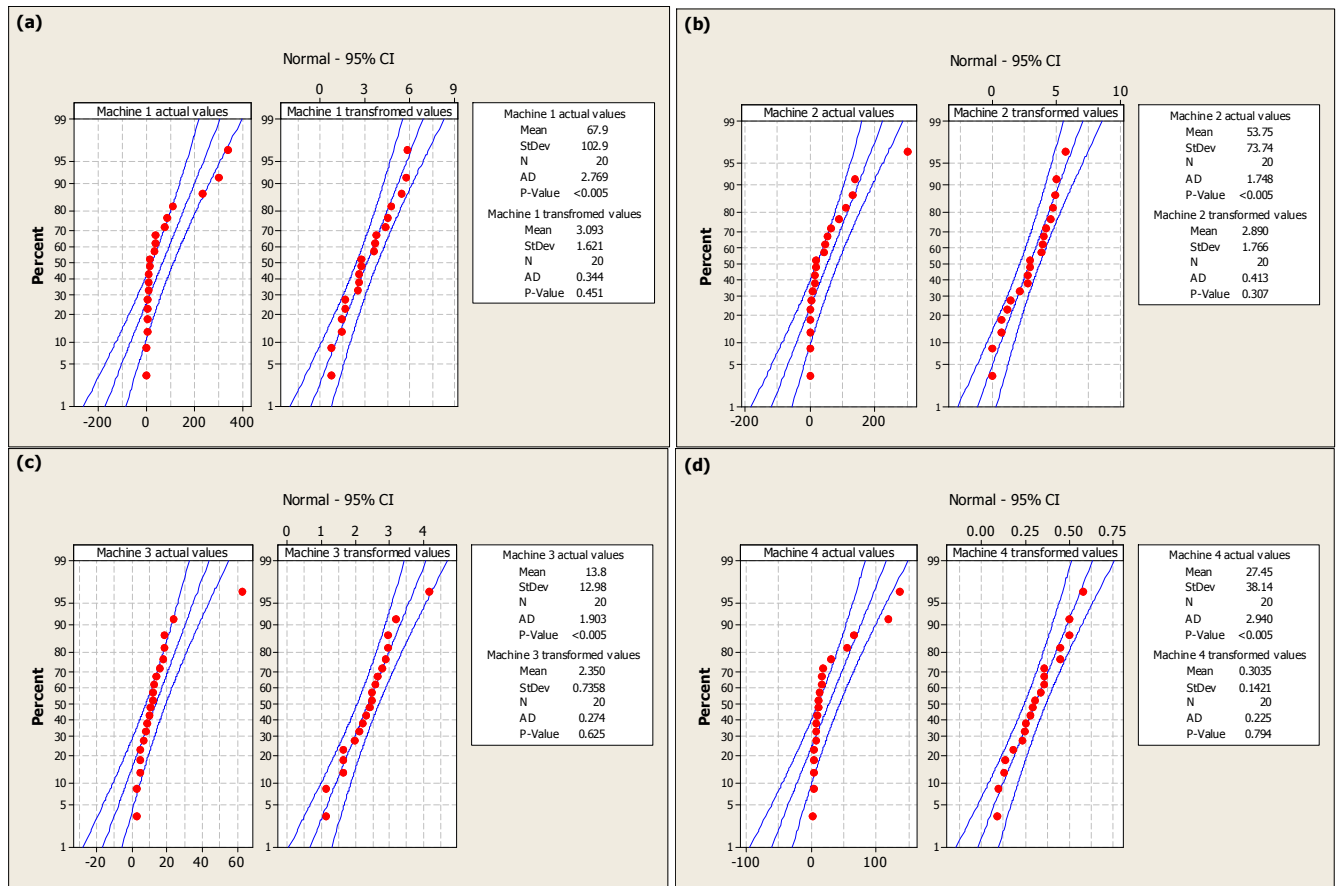


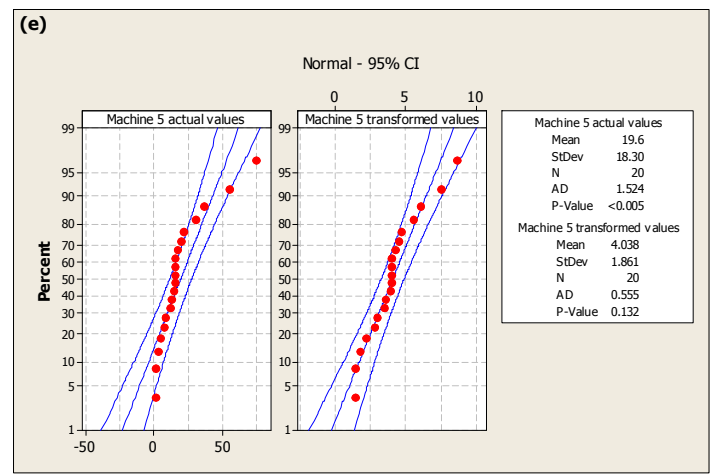

Figure 5 Probability plots of actual and transformed values; (a) machine 1, (b) machine 2, (c) machine 3, (d) machine 4, (e) machine 5

Table 2 is the summary of the twenty consecutive observations or actual values (AVs) on each machine and the Box-Cox transformed value (TV) for each observation, using the rounded $\lambda$ values. The transformed values were used to plot the Individuals control chart.

Table 2. Summary of observed and transformed data for each machine.

\begin{tabular}{|c|c|c|c|c|c|c|c|c|c|c|}
\hline \multirow{2}{*}{$\begin{array}{l}\text { Obsv. } \\
\text { No. }\end{array}$} & \multicolumn{2}{|c|}{ Machine 1} & \multicolumn{2}{|c|}{ Machine 2} & \multicolumn{2}{|c|}{ Machine 3} & \multicolumn{2}{|c|}{ Machine4 } & \multicolumn{2}{|c|}{ Machine 5} \\
\hline & $\begin{array}{l}\text { AV } \\
\text { (min.) }\end{array}$ & $\mathrm{TV}$ & $\begin{array}{l}\text { AV } \\
(\min )\end{array}$ & $\mathrm{TV}$ & $\begin{array}{l}\text { AV } \\
(\mathrm{min})\end{array}$ & $\mathrm{TV}$ & $\begin{array}{l}\text { AV } \\
\text { (min) }\end{array}$ & $\mathrm{TV}$ & $\begin{array}{l}\text { AV } \\
(\mathrm{min})\end{array}$ & TV \\
\hline 1 & 234 & 5.45 & 141 & 4.95 & 19 & 2.94 & 13 & 0.28 & 16 & 4.00 \\
\hline 2 & 340 & 5.83 & 1 & 0.00 & 14 & 2.64 & 55 & 0.13 & 16 & 4.00 \\
\hline 3 & 2 & 0.69 & 49 & 3.89 & 5 & 1.61 & 12 & 0.29 & 9 & 3.00 \\
\hline 4 & 303 & 5.71 & 45 & 3.81 & 10 & 2.30 & 4 & 0.50 & 37 & 6.08 \\
\hline 5 & 4 & 1.39 & 4 & 1.39 & 8 & 2.08 & 31 & 0.18 & 56 & 7.48 \\
\hline 6 & 89 & 4.49 & 1 & 0.00 & 11 & 2.40 & 8 & 0.35 & 31 & 5.56 \\
\hline 7 & 5 & 1.61 & 2 & 0.69 & 19 & 2.94 & 9 & 0.33 & 22 & 4.69 \\
\hline 8 & 15 & 2.71 & 15 & 2.71 & 13 & 2.56 & 17 & 0.24 & 3 & 1.73 \\
\hline 9 & 5 & 1.61 & 133 & 4.89 & 7 & 1.94 & 4 & 0.50 & 5 & 2.23 \\
\hline 10 & 4 & 1.39 & 111 & 4.71 & 12 & 2.48 & 19 & 0.23 & 16 & 4.00 \\
\hline 11 & 13 & 2.57 & 18 & 2.89 & 3 & 1.10 & 5 & 0.45 & 16 & 4.00 \\
\hline 12 & 41 & 3.71 & 15 & 2.71 & 3 & 1.10 & 3 & 0.57 & 20 & 4.47 \\
\hline 13 & 13 & 2.56 & 301 & 5.71 & 16 & 2.77 & 5 & 0.45 & 12 & 4.46 \\
\hline
\end{tabular}




\begin{tabular}{lllllllllll}
\hline 14 & 109 & 4.69 & 89 & 4.49 & 5 & 1.61 & 136 & 0.08 & 8 & 2.82 \\
15 & 15 & 2.71 & 64 & 4.16 & 24 & 3.17 & 8 & 0.35 & 13 & 3.60 \\
16 & 2 & 0.69 & 3 & 1.10 & 63 & 4.14 & 66 & 0.12 & 2 & 1.41 \\
17 & 12 & 2.48 & 8 & 2.08 & 9 & 2.19 & 119 & 0.09 & 18 & 4.24 \\
18 & 38 & 3.64 & 54 & 3.99 & 18 & 2.89 & 8 & 0.35 & 15 & 3.87 \\
19 & 79 & 4.37 & 2 & 0.69 & 5 & 1.61 & 16 & 0.25 & 2 & 1.41 \\
20 & 35 & 3.55 & 19 & 2.94 & 12 & 2.48 & 11 & 0.30 & 75 & 8.66 \\
\hline
\end{tabular}

Obsv: observation; AV: actual value; TV: transformed value

Control limits for the Shewart Individuals chart are calculated using the following equations (Montgomerry, 2009 pp. 260)

$$
\begin{aligned}
& \mathrm{UCL}=\bar{X}+3 \frac{\overline{M R}}{d_{2}} \\
& \text { Centre line }=\bar{X} \\
& \mathrm{LCL}=\bar{X}-3 \frac{\overline{M R}}{d_{2}}
\end{aligned}
$$

where UCL and LCL are the upper and lower control limits respectively, $\bar{X}$ is the average or mean of the individual observations, $\overline{M R}$ is the average of the moving range measurements and $\mathrm{d}_{2}=1.128$ (this is a given constant if the moving range is between two individual observations). The moving range $\mathrm{MR}$ is the difference between two consecutive observations.

The initial control limits (CLs) are derived from the first batch of data and are not changed arbitrarily. They remain valid unless there is a significant and sustained process shift that warrants a recalculation of the CL (Schaeffers and Stephen, 2010). Analysing a control chart requires knowledge of the process, and proficiency in distinguishing between incontrol (common and predictable causes) and out-of-control (special and unpredictable) causes of variation in a process. The Western Electric zone rules are typically used in most control charts to detect out-of-control conditions in a process, exposing unusual patterns within the CL and observations that lie outside of the CL. If variations are truly common and random, then observation points should have an equal number of data that lie above and below the centre line. Application of the zone rules needs to be performed with care so that the control chart is not excessively sensitive. Most researchers recommend the use of two basic rules or zone tests to signify an out-of-control point or 
pattern: any point falling outside the CL and; eight consecutive points falling on the same side of the centre line (Noskievičová, 2013).

\section{Experimental results of the scheduling data for each machine}

The IX control charts of the transformed data for the individual observations are shown in Figure 6(a) to (e). Each chart was analysed for out-of-control data points and patterns following the two zone tests chosen in the previous section.

The probability of an observation falling outside the CLs when a process is unchanged and performing as normal is $0.27 \%$ (Fasting, 2009). So in effect, any data point outside the CLs is unlikely to result from normal variations within the process.

The scheduling data for machines 1 and 2 show some similarities. An analysis of the IX charts, Figure 6(a) and (b) shows the data points are randomly and evenly distributed above and below the centre line. In addition they all lie within the control limits calculated from the data set. Although there are no out-of-control data points or patterns, the actual values in columns 2 and 4 in Table 2, comprise many extreme variations. For instance, in machine 1 , about nine or approximately $50 \%$ of the observations have variations that are above 30 minutes and six observations are above 100 minutes, of which two are above 300 minutes variation.
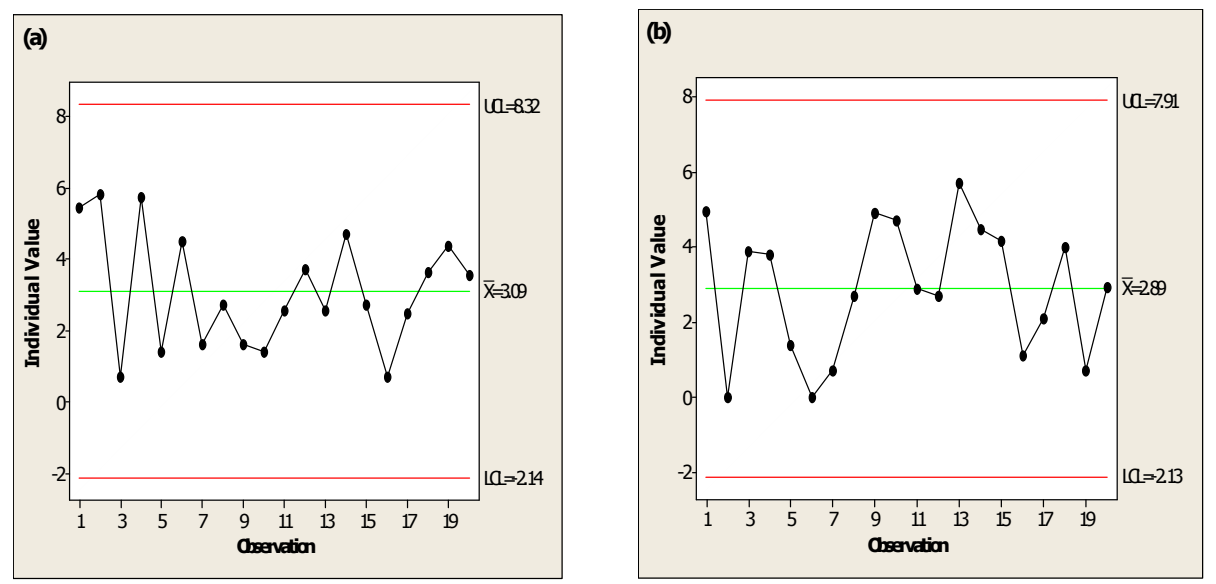

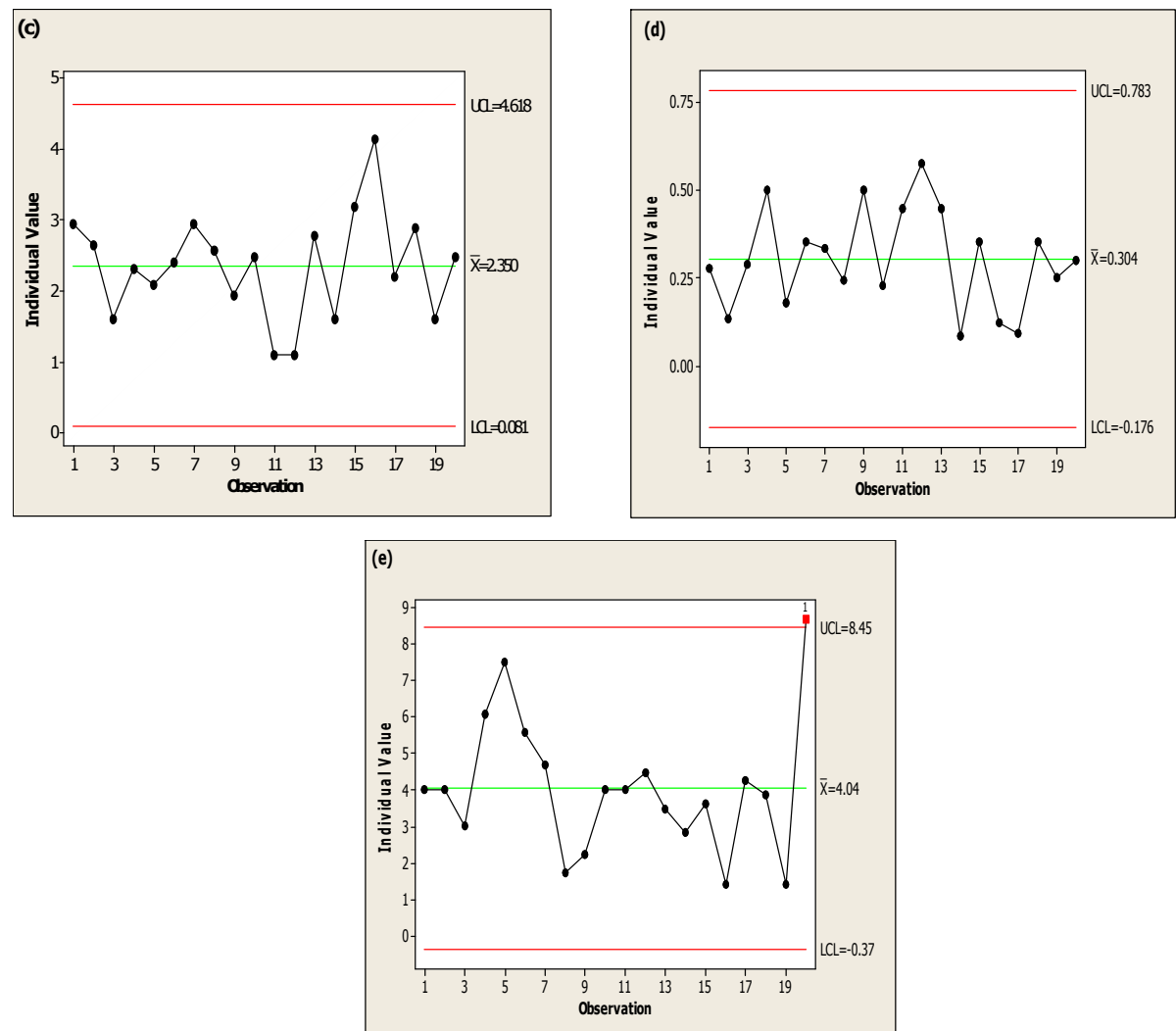

Figure 6. IX control charts for deviations of actual process time from scheduled process time: (a) machine 1, (b) machine 2, (c) machine 3, (d) machine 4 and (e) machine 5.

The scheduling process for this machine, and for machine 2, although in-control, will consistently and predictably return high variations if not checked. Upon investigation on the cause of the consistently high variations in the process times for these machines, it was found that the machine is of the first generation set of machines installed by the factory. The machines are old, require many manual operations, and when some jobs are mounted on the machine, a slower speed is sometimes used so that a high quality output is achieved and waste is minimised. For those variations that are above 50 minutes, it was found that the machine was stopped during processing because the job was awaiting materials from another section.

The scheduling data for machine 3 as shown in the IX chart Figure 6(c) and in the actual values in column 6 of Table 2, show that the scheduling process is in control, and the process variations are not as wide as those for machines 1 and 2. Upon further enquiries 
it was found that machine 3 processes the simplest and least complex types of jobs and is also a simple machine to operate and handle, and so the process variations are minimal from this machine.

In the IX chart for machine 4 scheduling data, Figure 6(d), the data points are evenly spread on either side of the centre line. No data points are outside of the control bands, and there are no out-of-control patterns as both tests passed. In addition, majority of the variations as exhibited in the raw data, are not too wide as shown in column 8 of Table 2. Of note, however, are two high variations for the $14^{\text {th }}$ and $17^{\text {th }}$ observations. These data points show up as points far below the centre or mean line because the data transformation is an inverse mathematical operation (see Table 1). In general, the scheduling process for machine 4 is not only in control, but experiences a narrow band of variations. Upon investigation, it was found that machine 4 is part of a new set of machines, and its operations are mainly automated, which explains its relatively stable process. The high process variations for this machine $\left(14^{\text {th }}\right.$ and $17^{\text {th }}$ data) were found to have resulted from minor machine break down due to operator error, because the operator was not fully familiar with its operations.

The scheduling data for machine 5 shows an out of control point for the $20^{\text {th }}$ observation, Figure 6(e). From the column containing the actual values, column 10, Table 2, the process variation of 75 minutes for the $20^{\text {th }}$ observation implies that the scheduling process for the machine is out of control at the $20^{\text {th }}$ job. An out of control point is not likely due to chance (Fasting, 2009). Although this value of 75 minutes is much lower than some values for machines 1 and 2 (which show an in-control process), the process mean for machine 5 is much lower than for machines 1 and 2 . In other words, the out-ofcontrol point for machine 5 will not be an out-of-control point if plotted for machine 1 or 2. Upon further investigation, it was found that the $20^{\text {th }}$ job was a new job, never processed in the factory. An approximate process time was scheduled for the job, based on similar jobs that had been processed on the machine in the past. The scheduled process time used was wrong and off target.

The above analysis (and the different Box-Cox data transformation values in Table 2) has shown that each machine exhibits its own unique set of data even though all five machines 
make the whole system and perform the same printing process. This supports the argument for the need to segregate processes into sub-processes for a better analysis of the entire system.

\section{Using SPC charts for performance measurement}

The experimental procedures outlined in the previous section were designed to introduce the use of SPC charts as a performance measurement tool. The SPC charts would need to be analysed within this context, and designed for continuous monitoring of the process in a PMS.

Setting targets is an important component in the design of an effective PMS. Using a PMS without targets will cause the PMS to fail in achieving its objectives. The targets are bounds or limits of expected performance. Using the IX charts in the previous section, the CLs easily act as performance targets for the scheduling process. The first set of charts are equivalent to the Phase I stage of any SPC methodology, and the CLs represent typical expectations of when SPC charts are initially introduced into a system. The presented CLs in the initial IX charts were used as beginning targets for each machine scheduling process performance measurement. If the CLs are taken as performance targets, the scheduling processes for machines 1 to 4 are performing within target, as there are no out-of-control points or patterns. The scheduling process for machine 5 performed below target, as there was an observation (the $20^{\text {th }}$ job) that fell outside of the CL. The adoption of a performance targets is the choice of the organization. To reset the performance targets in the system being studied will require that the CLs are recalculated and reset. Setting of CL discretionally will lead to Type I, and Type II errors. If the CLs are too wide the IX chart will fail to register under-performance and vice-versa (Noskievičová, 2013). Flaig (2010) proposed an action plan to take on when to recalculate CLs, Table 3, and this guide is proposed for recalculating CLs and setting realistic performance targets in the future. 
Table 3. When to recalculate control limits in a SPC chart (Flaig, 2010).

\begin{tabular}{lll}
\hline Search for Cause(s) & Corrective Action(s) & Recalculate Control Limits \\
\hline $\begin{array}{l}\text { 1. If the cause(s) is/are } \\
\text { not found }\end{array}$ & $\begin{array}{l}\text { Take no action but continue search } \\
\text { for cause(s) }\end{array}$ & $\begin{array}{l}\text { Do not recalculate the CL. } \\
\text { Instead, leave CL as it is. }\end{array}$ \\
$\begin{array}{l}\text { 2. If the cause(s) is/are } \\
\text { found and the process } \\
\text { has improved }\end{array}$ & $\begin{array}{l}\text { 1. If the process improvement can } \\
\text { be locked in, then adopt the process } \\
\text { change }\end{array}$ & $\begin{array}{l}\text { 1. Recalculate the CL using the } \\
\text { CL from the improved } \\
\text { data/process. }\end{array}$ \\
& $\begin{array}{l}\text { 2. If the process improvement } \\
\text { cannot be locked in, then do not } \\
\text { adopt the process change. }\end{array}$ & $\begin{array}{l}\text { 2. Do not recalculate CL, as this } \\
\text { creates problems for the SPC. }\end{array}$ \\
$\begin{array}{l}\text { 3.If the cause(s) is/are } \\
\text { found and the process } \\
\text { has degraded, } \\
\text { permanently. }\end{array}$ & $\begin{array}{l}\text { 1. If the cause(s) can be eliminated, } \\
\text { then fix the problem }\end{array}$ & $\begin{array}{l}\text { 1. Do not re-compute the CL, as } \\
\text { the process should have now } \\
\text { returned to its previous } \\
\text { controlled state. }\end{array}$ \\
& $\begin{array}{l}\text { 2. If the causes(s) cannot be } \\
\text { eliminated, then problem cannot be } \\
\text { fixed }\end{array}$ & $\begin{array}{l}\text { 2. Recalculate CL using data } \\
\text { taken from the process after the } \\
\text { change has occurred. }\end{array}$ \\
\hline
\end{tabular}

\section{Discussion of the method}

By adopting a case study approach to a real life environment, this research has been able to explore an area that is rarely researched. The experimental methods contained in this research used SPC charts to design a PMS for a scheduling process. The scheduling performance metric that was studied was process time for each job order. The process parameter adopted for the performance metric was the deviation of actual process time from scheduled process time. By applying SPC charts to this quality parameter, the process performance was measured and a Process Performance Measurement System (PPMS) was introduced. It was found that the scheduling process for one of the machines in the system under investigation was out-of-control, and so the performance of the scheduling process for that machine was poor.

Our proposed methodology works with minimal assumptions thereby improving its applicability in many other scheduling situations. One assumption that we relied upon is that a job cannot me moved from one machine to another once processing has commenced. This assumption may be challenged in real life and if it is not a regular 
occurrence, such jobs may be expunged from the observed data. However if it is a regular occurrence then the observed data should be divided between the machines.

The validation of this research is embedded in the methodology that was adopted. By recording and plotting every observation on the IX chart, we eliminated errors that are caused by inappropriate sampling methods or erroneous data. By studying the scheduling performance for five machines we ensured the method was repeated five times thereby improving our confidence in the chosen approach. This proves that the methodology cab be extended to measure schedule error in other processes within the entire system. The check for normality and correlation added reliability to our IX charts. A different BoxCox transformation was obtained for each machine as the statistical software that was used chooses an optimal mathematical operation to get the highest $p$ value. The differences in the Box-Cox transformation used for each machine is explainable as each data represents a different sub-process

The frequency of measuring the performance of a system is the prerogative of the organization, but largely depends on dynamics of the process being measured, the objectives of the PMS and the costs of measuring performance. The experimental methods outlined in the previous section describes the setting of targets using the CLs calculated from the first set of data, and measuring the schedule performance for each job, thereafter, becomes easy. Every subsequent job observation is extended and plotted on the IX chart developed from the first set of data, using the same CLs. The AVs of the subsequent observations are transformed using the same $\lambda$ that was used to calculate the CLs. The CLs remain unchanged unless a process shift has occurred and which can be proved to have resulted from an improvement or degradation of the process.

Finding an optimal schedule is one task and measuring how that optimal schedule has performed in reality is another task. With our method, a scheduler can monitor how an optimal schedule has performed on the shop floor, study the variations between plans and outcomes and seek ways of minimizing schedule error and check if process improvements have been effective. Our method helps to ensure the system is consistently in control and therefore predicable for planners to work with. 
Decision making needs to be based on facts and the experimental methods contained in this case study ensures that. PPMSs should gather performance-relevant information; compare current data with past or target; and communicate the results with other stakeholders. The use of SPC charts corroborates this statement. Good PPMSs should communicate the causes of any performance problems. An inaccurate schedule time was used for a job on Machine 5, which caused the scheduling process to be out-of-control in the $20^{\text {th }}$ observation, causing the process to underperform. If we assume that variations above 30 minutes are unacceptable, we can plot a Pareto chart for causes of variations above 30 minutes, see Figure 7 . Clearly approximately $85 \%$ of the causes of variations arise from three main areas; machine stopped and awaiting raw materials: avoidable temporary machine breakdown: and inaccurate schedule time used. These causes of wide variations can be easily corrected, for example it is easy to ensure that a job is not scheduled unless the raw materials for the job are $100 \%$ available for the process.

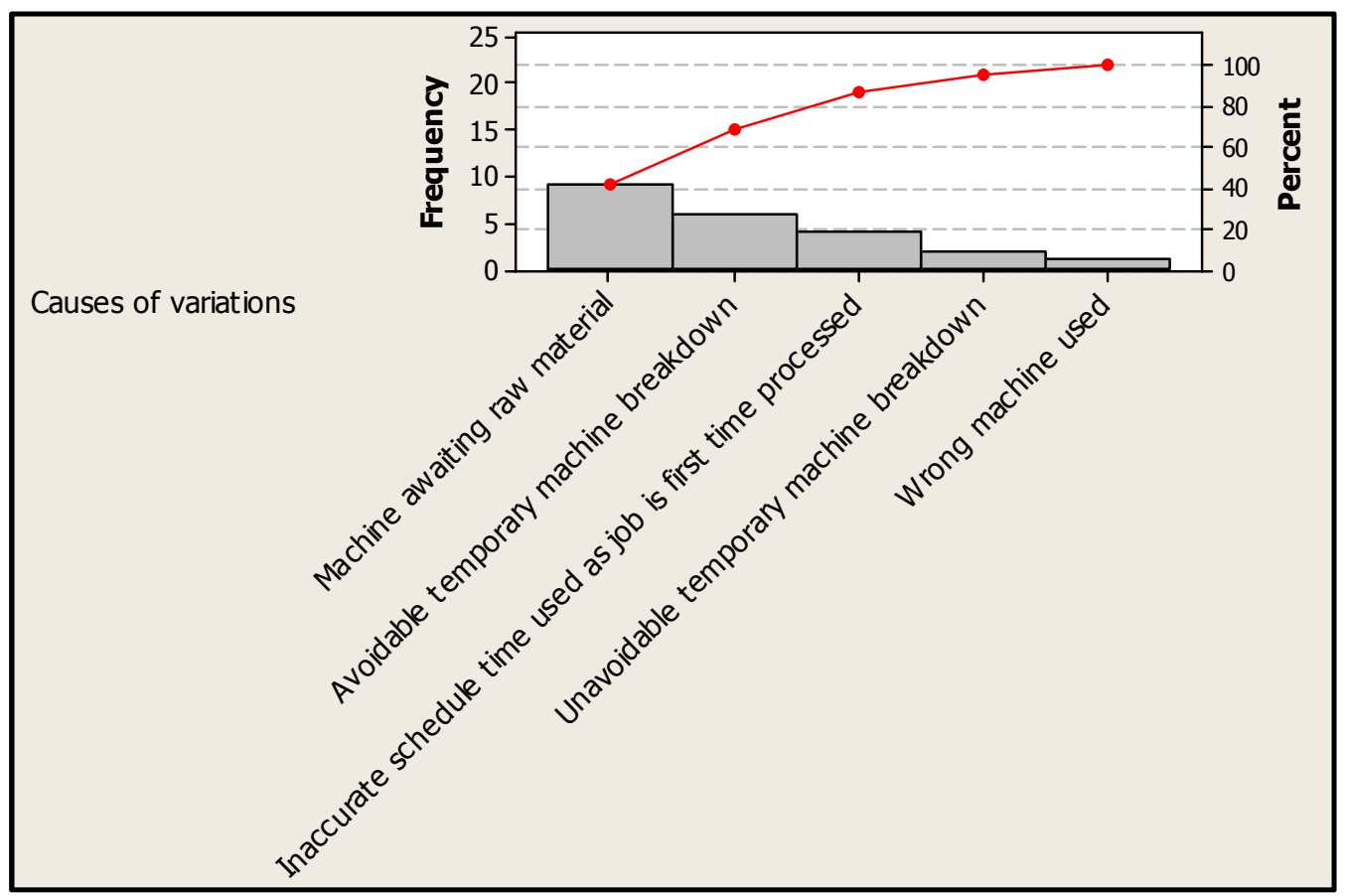

Figure 7. Pareto chart, showing the causes of variations that are above 30 minutes, for all the machines.

In addition, any first time job can be eliminated from the data set to eliminate causes of variations arising from wrong schedule time as evidenced in machine 5 out-of-control 
data. If done, process variations should fall within narrower CLs on the control charts, allowing an adjustment of the control limits as the process performance improves. By adopting the SPC chart approach we were able to seek out drawbacks to performance while also presenting the causes of the problems, with a view to eliminating them and improving performance. If we use the results from the Pareto analysis in Figure 7 as a basis and the main causes of variations above 30 minutes are eliminated, then majority of schedule error data will be no more than 30 minutes. The UCL and process mean will drop and performance targets can be made tighter. Our methodology therefore helps in setting realistic and achievable targets as the charts display the capability of the system.

\section{Conclusions}

Most studies on scheduling have focused on finding an ideal schedule for a set of job shop orders, but little work has been done to measure the performance of the optimal schedule. The methodology outlined in this research paper provides an approach to effectively and continuously measure the performance of a schedule. Statistical Process Control charts monitor variations within a process and are widely used in performance management systems. By applying the Individuals Control chart to the scheduling system for a job shop, we were able to use the control limits of the chart as performance targets while the variations in the schedule could be monitored to seek ways of eliminating or reducing the variations. The method when applied would provide more accurate schedules, which improve organizational planning.

Our proposed methodology forms a basis for applying control charts in the measurement of schedule performance. In our analysis we did not encounter data correlation, which is moderate to high for most job shop schedule performance metrics, such as flow time and works-in-process. Machine utilization which is another schedule performance metric may exhibit high correlation across sub-group data. In our study, we proposed a course of action to be taken when the data are correlated i.e. fitting a time series model to the data, and that is the only alteration we envisage, to our proposed method. The challenge of fitting an appropriate time series model to the correlated data and applying our proposed method opens an area for further research. This would expand the application of our 
approach to virtually all schedule performance metrics with immense benefits to research and practitioners.

\section{References}

Apley, D.W. (2002) "Time series control charts in the presence of model uncertainty", Journal of Manufacturing Science and Engineering Vol. 124, pp. 891-898.

Buthmann, A. (2010) "Making data normal using Boc-Cox power transformation", www.isisxsigma.com/tools-templates/normality/making-data-normal-using-boxpower-transformation/ (accessed 6 June 2013).

Chen, M.-C., Su, C.-T., Hsu, C.-C. and Liu, Y.-W. (2005) "Data transformation in SPC for semiconductor machinery control: a case of monitoring particles" International Journal of Production Research Vol. 43, pp. 2759-2773.

Coleman, S.Y., Arunakumar G., Foldvary, F. and Feltham, R. (2001) "SPC as a tool for creating a successful business measurement framework" Journal of Applied Statistics Vol. 28, pp. 325-334.

Della, R. (2000) “Harley rides high on SPC changes”, Quality Vol. 39, pp. 40-43.

De Snoo, C., Van Wezel, W. and Jorna, R.J. (2011) "The use of scheduling performance criteria in practice" Journal of Operations Management Vol. 29, pp. 181-19.

Fasting S. (2009) "Statistical process control part 2: using statistical process control to measure process improvement and patient flow", Australian Health Review Vol. 33 , pp. 412-422.

Flaig, J.J. (2010) "When to recalculate control limits", www.isixsigma.com/toolstemplates/control-charts/when-recalculate-control-limits/ (accessed 6 June 2013).

Fullerton, R.R. (2003) "Performance measurement and reward systems in JIT and nonJIT firms", Cost Management Vol. 17, pp. 40- 48.

Grygoryev, K. and Karapetrovic, S. (2005) "Tracking classroom teaching and learning: an SPC application”, Quality Engineering Vol. 17 pp. 405-418.

Henri, J. (2006) “Are your performance measurement systems truly performing?" CMA Management Vol. 80, pp. 31-35.

Kueng, P. (2000) "Process performance measurement system: a tool to support processbased organizations", Total Quality Management Vol. 11, pp. 67-85. 
MacCarthy, B.L. and Wasusri, T. (2002) "A review of non-standard applications of statistical process control charts", The International Journal of Quality \& Reliability Management Vol. 19, pp. 295-320.

MacCarthy, B.L. and Wasusri, T. (2001) "Statistical process control for monitoring scheduling performance- addressing the problem of correlated data", Journal of the Operational Research Society Vol. 52, pp. 810-820.

Montgomerry, D.C. (2009) Introduction to statistical quality control. $6^{\text {th }}$ ed. New Jersey: John Wiley \& Sons.

Morgan, C. and Dewhurst, A. (2007) "Using SPC to measure a national supermarket chains' suppliers' performance" International Journal of Operations \& Production Management Vol. 27, pp. 874-900.

Neely, A.D., Adams, C. and Kennerly, M. (2012) "The performance prism: The scorecard for measuring and managing stakeholder relationships", In: Striteska $\mathrm{M}$ and Svoboda O. Survey of performance measurement systems in Czech companies. Economie a Management, Vol. 2, pp. 68-84.

Noskievičová, D. (2013) “Complex control chart interpretation”, International Journal of Engineering Business Management, Vol. 5, pp. 1-7.

Ouelhadj, D. and Petrovic, S. (2009) "Survey of dynamic scheduling in manufacturing systems", Journal of Scheduling Vol. 12, pp. 417-431.

Rungasamy, S., Jiju, A. and Ghosh, S. (2002) "Critical success factors for SPC implementation in UK small and medium enterprises: some key findings from a survey" The TQM Magazine Vol. 14, pp. 217-224.

Schaeffers, M., and Stephen, C. (2010) "Recalculating control limits", www.isixsigma.com/tools-templates/control-chart/recalcualting-control-limits/ (accessed 6 June 2013).

Slavin, V. (2006) Improper use of control charts: Traps to Avoid, www.seicmu/library/assets/slavin.pdf (accessed 30 May 2013).

Stanislava, S. (2012) "Identification of data content based on measurement of quality performance", Ekonomie a Management Vol. 1, pp. 128-138.

Wadsworth, H.M., Stephens, K.S. and Godfrey, A.B. (2002) "Evaluating environmental performance using statistical process control techniques", European Journal of Operational Research Vol. 139, pp. 68-83.

Wasusri, T. and MacCarthy, B. (2001) "Using SPC chart techniques in production planning and scheduling: two case studies", In: POM conference on operations planning, scheduling and control, Florida, USA, 30 March-2 April 2001. 
2014-03-14

\section{Schedule performance measurement} based on statistical process control charts

\section{Oleghe, Omogbai}

Inderscience

Omogbai Oleghe and Konstantinos Salonitis. Schedule performance measurement based on statistical process control charts. International Journal of Engineering Management and Economics, 2014, Vol. 4, No. 3/4, pp194-212

http//dx.doi.org/10.1504/JJEME.2014.066940

Downloaded from Cranfield Library Services E-Repository 